\title{
Heuristik der Furcht
}

\section{Endlosigkeit als kontrafaktische Unterstellung - oder: selbst Hans Jonas hofft, aus Prinzip}

Hartmut von Sass

Look up here, I'm in heaven I've got scars that can't be seen

I've got drama, can't be stolen

Everybody knows me now

Look up here, man, I'm in danger

I've got nothing left to lose I'm so high, it makes my brain whirl Dropped my cell phone down below Ain't that just like me?

By the time I got to New York I was living like a king Then I used up all my money I was looking for your ass

This way or no way You know I'll be free Just like that bluebird Now, ain't that just like me?

David Bowie, Lazarus (2016)

\section{I. »Für immer« - never, ever!}

Erst Lemmy Kilmister, nun David Bowie. Ich weiss gar nicht recht, welcher Tod mich mehr getroffen, berührt hat, obwohl ich weder Motörhead-Fan bin, noch Ziggy-Stardust-Jünger sein möchte. Ich muss sogar zugeben, dass ich sie beide auf ganz unterschiedliche Weise nicht habe ernst nehmen wollen; sie blieben mir eigentümlich entrückt, ja fern.Vielleicht lag das genau an dem, wofür sie ikonisch geworden sind und nun, im elegisch-enthusiastischen Nachruf, ge- 
rühmt werden: Im einen Fall könnte es die verspielte Ehrlichkeit eines militäraffinen Devotionalien-Sammlers sein, der sich OrangeWodka, Prostituierten und Computerspielen (wohl in der Reihenfolge) hingab, wenn er nicht in dieser sagenhaften, ganz schwarzen Montur auf der Bühne stand, um jedes Konzert mit den Satz zu beginnen: »We are Motörhead, and we're playing rock'n roll«. (Dieses Proömium lebte weniger von seiner ritualhaften Wiederholung als von der gezielten Untertreibung, denn was dann folgen sollte, war bester heavy metal). Und im anderen Fall, dem Bowies, war es diese glamourhafte Wandlungskapazität, die ihn immer woanders sein liess und nun als gesellschaftsveränderndes Statement angeeignet, vielleicht gar vereinnahmt wird. Der Mann aus Brixton, der eigentlich ganz anders hiess (und also selbst vor seinem Namen flüchtete), gab allen, die anders waren, die rekursive Rechtfertigung als exemplum mit oder steckte jenen, die anders sein wollten, Anleitungen zu, wie diese Alterität aussehen könnte. Kilmister sah immer gleich aus, Bowie stets anders. Beides, so ist zu vermuten, waren Kostüme. Und meine Befürchtung ist, dass sie echt waren. So blieben sie auf Distanz.

Für unser Thema ist es nun nicht ohne Relevanz, sich die Modi der Rückversicherung anzusehen, die in der Berichterstattung vom Ableben beider, vor allem Bowies, kursieren. Während man bei Kilmister eher mit Ver- und Bewunderung feststellen musste, dass er trotz aller Eskapaden, die ja gerade latent oder gar gewollt mit dem eigenen Ende spielten, noch immer da war, bewegte sich Bowie tatsächlich in einer eigenartigen space oddity. Mit Ableben und Ende hatte dieser Heros kaum etwas zu tun; dafür schien er zu jung, ewig jung, auch zu nobel - "the thin white duke "-, und es gelang ihm, ohne in den "club twenty-seven" aufgenommen zu werden, diesen Status zu konservieren. Nun heisst es plötzlich: »Der Krebs hat sein viertes, sechstes, $\mathrm{x}$-tes Comeback zunichte gemacht. David Bowies Werk für die Ewigkeit wird er nicht kleinkriegen. Niemals. «1

Familienähnliche Befunde liessen sich anfügen; sie alle verarbeiten die Diskrepanz zwischen Ende und dessen Dementi - "niemals" mit derTrennung von Person und Werk. Und diese Differenz nimmt nun wiederum zwei unterschiedliche Formen an, die uns näher ans Theologische führen: Entweder verhält sich das Dual von persona und opus wie die Schale zum Kern - so schlimm der Tod auch sein mag, die Essenz lebe weiter, sodass die Person in ihr eigenes Geschöpf aufsteigt, um im Werk für immer von nochmaligen Toden

\footnotetext{
1 http://www.zeit.de/kultur/musik/2016-01/david-bowie-pop-nachruf (zuletzt angesehen irgendwann in der Nacht vom 15. zum 16. Januar 2016).
} 
unbehelligt (oder diesen gerade ausgesetzt) zu sein. ${ }^{2}$ Oder aber das Dual spannt sich zwischen Vergessen und Rezeption auf, wobei Bowie - man blicke bitte nochmals auf die Eingangslyrik - die Auferstehung in seine Fan-Gemeinde vorwegnimmt, indem er zwischen Studio und Sterbebett dichtet:

Look up here, I'm in heaven

I've got scars that can't be seen

I've got drama, can't be stolen

Everybody knows me now.

Bowie erscheint hier als Jesus und Lazarus in einem, weil er selbst die Bedingungen dafür geschaffen hat, auferweckt zu werden. Allerdings kommen beide skizzierten Varianten besagter Diskrepanz dann doch noch einmal zueinander, weil selbst das Werk als Kern die Rezeption durch eine Audienz benötigt. Doch auch die ist, wie wir nur zu gut wissen und womöglich noch besser verdrängen, prekär bis brüchig. Auch die Subjekte der Aneignung revidieren die Auferstehung ins Ekklesiale; denn für niemanden gilt jenes »Für-Immer«. At some point no one knows you anymore. ${ }^{3}$

Nun ein Sprung, der keiner ist: Wenn wir in der Welt der Personen und Schalen, dem Vergessen und Gedenken keine Endlosigkeit finden, dann vielleicht in der Sprache über all das. Wie steht es mit dem Wahrheitswert der Propositionen, zum Beispiel, dass es ein "Für-Immer« niemals gibt? Konkret: Sind Ereignisse, die geschehen werden, schon jetzt wahr (also die Sätze über sie)? So fragt Aristoteles. ${ }^{4}$ Ist ein Sachverhalt, bevor er entdeckt wurde, wahr gewesen? So fragt Richard Rorty. ${ }^{5}$ Und können Kontrafakten, die wir durch Imagination und Phantasie annehmen und abwägen, wahr sein? So fragt Olaf Müller. ${ }^{6}$ (Ich weiss - Aristoteles/Rorty/Müller: ein sa-

\footnotetext{
2 Paul Tillichs Bestimmung eines "protestantischen Prinzips" bei gleichzeitiger Erwägung, der positiv-geschichtliche Protestantismus könnte an sein Ende kommen, folgt derselben Architektur - mit analogen Problemen. Dazu Ulrich Barth, Protestantismusverständnis und Kulturtheorie. Kontinuität und Wandel im Werk Tillichs, in: ders., Kritischer Religionsdiskurs, Tübingen 2014, 408-430, 425.

3 Frage der Herausgeber: „Denkst du, das gilt auch für Jesus? « Natürlich! Aber das ist kein spekulatives Urteil, sondern es geschieht ja längst; und warum sollte diese Entwicklung davon ausgeschlossen sein, sich irgendwann zu vvollenden??

4 Vgl. Metaphysik, Buch IX, 10.

5 Dazu Richard Rorty, Kontingenz, Ironie und Solidarität. Übersetzt von Christa Krüger, Frankfurt a.M. 1992, Kap. 1: "Kontingenz der Sprache«, bes. 24, 31, 49.

6 Siehe Olaf L. Müller, Chaos, Krieg und Kontrafakten. Ein erkenntnistheoretischer Versuch gegen die humanitären Kriege, in: Pazifismus. Ideengeschichte, Theorie und Praxis, hg. v. Barbara Bleisch/Jean-Daniel Strub, Bern/Stuttgart/Wien 2006, 223-263.
} 
genhaftes Dreigestirn).Vielleicht wäre also die Sprache, genauer: ihre wahren Präpositionen, Kandidaten für jenes »Für-Immer», nach dem wir suchen. Dazu müsste man die Zusatzthese durchbringen, nach der es Wahrheit ohne Träger, Wahrheitswerte also ohne Audienz gebe. Doch das ist selbst unter konservativen Theologen umstritten, die jenes Strittigsein allerdings gleich dogmatisch ausschlachten wollen. So baut Robert Spaemann seinen Gottesbeweis darauf, dass Wahrheit von jemandem gewusst werden müsse. Das, was gewesen sein wird, selbst wenn wir nicht mehr sein sollten, setze also jemanden voraus, der Träger jener Information bleibe. Aus dem Futurum II folge die Existenz solch eines Wesens, da sonst das propositional Wahre aufhöre, wahr zu sein, was nicht sein dürfe. Also existiert solch ein Wesen, das wir Gott nennen. ${ }^{7}$

Lässt man sich auf solch abenteuerliche Manöver nicht ein, könnte es geschehen, dass es bei der Einsicht bleiben muss, Wahrheit sei an ein Publikum, für das etwas wahr ist, gebunden. Dieser kleine Exkurs führt dann nur zurück auf das, was bereits zu Kilmister und Bowie vorgetragen wurde: Ihr »Für-Immer" ist an eine ihr Euvre aneignende, i.e. mitsingende Gemeinde gebunden, sodass wir - wer wäre überrascht? - auf's Endliche zurückgeworfen sind.

$\mathrm{Ob}$ das eine schlechte Nachricht, ein Gegen-Evangelium, sein muss, ist damit noch keineswegs ausgemacht. Der Mensch wolle, dass Gott nicht sei und er selbst Gott sei, meint bekanntlich Luther. Ewig wolle er, der Sünder, sein, dessen Tod nur der "Sünde Sold" bilde (Röm 6,23 - in der Sprache von Kilmister und Bowie: "For the wages of $\sin$ is death «). Ohne Sünde also kein Tod, Sünde als die Revision eines ursprünglichen "Für-Immer«? Calvin fügt dem mit Blick auf das quasi-apokalyptische Zeugnis (etwa Mt 25) eine eigene Note hinzu: Nicht die Sünde mache ein Ende, sondern Gottes ewiger Ratschluss begründe ein doppelt kodiertes »Für-Immer» im unversöhnlichen Entweder/Oder ewiger Erwählung und ebenso ewiger Verdammnis. ${ }^{8}$ Einmal abgesehen davon, wie man zu dieser Eschatologie steht, ${ }^{9}$ bringt sie ein neues Element ins Spiel, ein Element, das uns gleich noch genauer beschäftigen wird.

\footnotetext{
7 So Robert Spaemann, Der letzte Gottesbeweis. Mit einer Einführung in die großen Gottesbeweise und einem Kommentar von Rolf Schönberger, Pattloch 2007, bes. 31 .

8 Dazu Johannes Calvin, Unterricht in der christlichen Religion. Institutio Christianae Religionis. Nach der letzten Ausgabe von 1559 übersetzt und bearbeitet von Otto Weber. Im Auftrag des Reformierten Bundes bearbeitet und neu herausgegeben von Matthias Freudenberg, Neukirchen-Vluyn ${ }^{2} 2009$, Buch III, Kap. 21-24.

9 Man kann dieses Lehrstück (mit Weber und Troeltsch) auch als Entlastungsstrategie von jeglicher Werkgerechtigkeit lesen; dazu Christian Link, Prädestination und
} 
Nähern wir uns dem, indem wir einen kurzen Blick zurückwerfen: Calvin scheint eine schon zuvor latente Traditionslinie prominent zu machen, die fragen lässt, wie wir vorweggenommene Zukunftsszenarien für unser gegenwärtiges Leben fruchtbar machen können - und sollen. Das heisst, es wird gerade nicht - im Gefolge von Jonathan Swift, G.B. Shaw und Queen - gefragt: who wants to live forever? Vielmehr wird vorgestellt, dass das derart Gefragte tatsächlich wahr wäre! - Was folgte aus dieser »schlechten (weil nur dauernden) Unendlichkeit«? Nietzsche leitete daraus in einem verspäteten Atomismus der Lehre von der ewigen Wiederkehr den existenziellen Imperativ $\mathrm{ab}$, das Leben so zu führen, dass man wollen können muss, dass es sich in der Tat ewig wiederhole. ${ }^{10}$ Nietzsche sagt nicht, dass es so sei, sondern nur (aber immerhin), dass wir unterstellen sollten, jenes Szenario entspreche der Wahrheit - mit eminenten Konsequenzen für unseren Lebensvollzug im Hier und Jetzt.

Wir haben mit Kilmister und Bowie begonnen und sind über Aristoteles (und einige seiner Nachfolger) sowie über die Reformatoren zu deren Erben Nietzsche gelangt. Nirgendwo war ein "Für-Immer» aufzufinden, aber ohne Hintersinn und ergebnislos ist unsere tour d'horizon (oder de force?) keineswegs geblieben. Denn sie führte uns zu einem Moduswechsel in der möglichen Betrachtung, sodass es gerade im Angesicht des Scheiterns aller Versuche, die Endlichkeit $\mathrm{zu}$ überspringen, $\mathrm{zu}$ überlisten, sie theologisch $\mathrm{zu}$ suspendieren etc. pp., darauf ankommen könnte, ihr Gegenteil kontrafaktisch zu unterstellen. Jene alte Orts- und Perspektivenangabe, alles sub specie aeternitatis anzuschauen, erhält hier neues Kolorit. Der nietzscheanische Imperativ - oder ist es ein Invitativ? - wird nun umkodiert: Es geht nicht mehr um die eigene zirkelhafte Ewigkeit, sondern es könnte darum gehen, von der Ewigkeit der Menschheit auszugehen - und zu fragen, was gegenwärtige Entscheidungen im Blick auf Gemeinschaft und deren Erhaltung mit sich bringen, zumal sie sich als nicht revidierbar erweisen könnten.

Eben dieser kontrafaktischen Unterstellung eines »Für-Immer» - die weit mehr ist als ein blosses >Als-Ob - wird im Folgenden nachgedacht. Ewigkeit wird hier nicht präsentisch als Intensivierung der Zeit und das Zusammenkommen der drei temporalen Modi

Erwählung, in: ders., Prädestination und Erwählung. Calvin-Studien, NeukirchenVluyn 2009, 33-54, 37.

${ }^{10}$ Alles Nötige (und mehr) bei Simon Springmann, ,Ring der Ringe`, Rad des Seins: Die Ewige Wiederkunft des Gleichen - kosmologischer Zirkel und existenzieller Imperativ?, in: Regress und Zirkel. Figuren prinzipieller Unabschließbarkeit: Architektur - Dynamik - Problematik, hg. v. Stefan Berg/Hartmut von Sass, Hamburg 2016 (im Druck). 
gleichsam entschärft, ${ }^{11}$ sondern ganz im Futurischen belassen. Nun also wird die Gegenwart vor das Tribunal der als ewig angenommenen Zukunft gezogen.

\section{Hans Jonas und sein »tractatus technologico- ethicus $^{12}$}

Um den Furor in Hans Jonas' Das Prinzip Verantwortung nicht als rein hyperbolische Geste verfrüht abzutun, damals, vielleicht auch heute, muss man sich kurz die historischen und geopolitischen Umstände vergegenwärtigen. 1979 erschienen und damit auf einem der Höhepunkte militärischer Aufrüstung, nuklearer Bedrohung, west-östlicher und also dualer Abschreckung, aber auch wirtschaftlichen Erfolgs und technischer Machbarkeit verweist Jonas auf die ungeheuren Ambivalenzen jener Entwicklung. Gleich im ersten Satz heisst es:

»Der endgültig entfesselte Prometheus, dem die Wissenschaft nie gekannte Kräfte und die Wirtschaft den rastlosen Antrieb gibt, ruft nach einer Ethik, die durch freiwillige Zügel seine Macht davor zurückhält, dem Menschen zum Unheil zu werden.« (7)

Wenig später fügt er hinzu:

"Das unvermeidlich sutopische Ausmaß moderner Technologie führt dazu, daß der heilsame Abstand zwischen alltäglichen und letzten Anliegen, zwischen Anlässen für gewöhnliche Klugheit und Anlässen für erleuchtete Weisheit stetig schrumpft." (54)

Jonas nimmt hier eines der Hauptthemen aus Heideggers Spätphilosophie auf. ${ }^{13} \mathrm{Im}$ Gegensatz zu seinem Lehrer bestimmt er die Technik aber nicht als neuzeitliche Entfaltung einer sich von

\footnotetext{
${ }^{11}$ Vgl. Eberhard Jüngel, Paulus und Jesus. Eine Untersuchung zur Präzisierung der Frage nach dem Ursprung der Christologie, Tübingen (1962) ${ }^{5} 1979,265 f$.

${ }^{12}$ So nennt Hans Jonas sein Hauptwerk: Das Prinzip Verantwortung. Versuch einer Ethik für die technologische Zivilisation [1979], Frankfurt a.M. ${ }^{5} 2015$, 9 (Seitenzahlen in Klammern beziehen sich auf diesen Text; abgekürzt PV.

${ }^{13}$ So stellt Heidegger fest: "Der Mensch ist auf dem Sprunge, sich auf das Ganze der Erde und ihrer Atmosphäre zu stürzen, das verborgene Walten der Natur in der Form von Kräften an sich zu reißen und den Geschichtsgang dem Planen und Ordnen einer Erdregierung zu unterwerfen. Derselbe aufständige Mensch ist außerstande, einfach zu sagen, was ist, was dies ist, dass ein Ding ist. / Das ganze Seiende ist Gegenstand eines einzigen Willens zur Eroberung." Ders., Der Spruch des Anaximander [1946], in: ders., Holzwege [1950], Frankfurt a.M. ${ }^{8} 2003,321-373,372$.
} 
jeher vollziehenden Selbstermächtigung. Vielmehr erkennt Jonas in der "technologischen Zivilisation" (so der Untertitel seines Werkes) gerade das ungeahnt Neue, auf das wir moralisch und ethisch, d.h. lebensweltlich und theoretisch nicht vorbereitet seien. Das macht den Befund umso dringlicher.

Im Gestus Günther Anders und Karl Jaspers verwandt, unterzieht Jonas die bisherige Ethik einer manifesten Kritik, da sie sich mit einem neuen Radius der technischen Potenz und einer schwer vorstellbaren Halbwertzeit menschlicher Entscheidungen anfreunden müsse. Der "Nahkreis des Handelns" (23) sei längst durchbrochen, sodass die anthropozentrische Ausrichtung der klassischen Ethik unbedingt aufzugeben sei - in dreierlei Hinsicht: Zum einen beklagt Jonas das etwa bei Kant vorherrschende "Verkleinern der kognitiven Seite sittlichen Handelns" (24). Damit weist Jonas die rationalistische Unterstellung ab, jeder sei prinzipiell fähig, sich zu einem reinen Vernunftwesen zu reduzieren in Absehung aller konkreten Umstände wie Biographie, Charakter, Bildung. Zum anderen, und für unser Thema gewichtiger, macht Jonas darauf aufmerksam, dass jede künftige Ethik eine Ethik des Künftigen sei, d.h. dass sie in sich die Einsicht zu integrieren habe, dass Täter und Betroffene nicht mehr identisch seien (vgl. 26). Verantwortung wird damit aus dem individuell-familiären "Nahkreis" entnommen, sodass die Implikate unserer Entscheidungen für folgende Generationen in einem Masse zu bedenken seien, das der Relevanz der Gegenwart im Hier und Jetzt gleichkomme. Und schliesslich habe die Ethik den Blick für's Humane gerade so beizubehalten, dass sie den Menschen dezentriere und als Teil der Natur begreife. Albert Schweitzers Ruf zur "Ehrfurcht vor dem Leben" klingt hier merklich nach, um in den Slogan der 1980er Jahre in den Zirkeln der (ost)deutschen Umwelt- und Friedensbewegung einzustimmen: "Bewahrung der Schöpfung«.Auch Jonas geht es also um dieVerletzlichkeit der Natur als einem ethischen Thema (26f. und 245), wobei die Begründungen unterschiedlich ausfallen; sie reichen von integrativen Mustern (der Mensch als Teil der Natur) bis hin zu anti-gnostischen und also monistischen Überzeugungen (der Eigenwert und die empfindsame Dynamik von Natur und Leben). ${ }^{14}$ Kurz: Jonas plädiert für eine dreifache Erweiterung des ethischen Fokus: anthropologisch im

${ }_{14}$ Dazu siehe Hans Jonas, Organismus und Freiheit. Ansätze zu einer philosophischen Biologie, Göttingen 1973; ders., Das Prinzip Leben. Ansätze zu einer philosophischen Biologie, Frankfurt a.M. 1997; ferner Benjamin Lazier, Overcoming Gnosticism: Hans Jonas, Hans Blumenberg, and the Legitimacy of the Natural World, in: Journal of the History of Ideas 64:4 (2003), 619-637. 
Blick auf dieVerantwortung des Menschen, temporal im Blick auf die Folgen unseres jetzigen Handelns, naturphilosophisch im Blick auf die Achtung des Lebens als ein Ganzes. Es ist insbesondere der zweite, zeitliche Aspekt, der im Prinzip Verantwortung bedacht wird. Bei aller mitunter harschen Kritik an Karl Marx (die dann vor allem dessen Rezeption im Utopismus Ernst Blochs betrifft; 348-390), schätzt ihn Jonas dafür, dass hier zum ersten Mal Verantwortung für geschichtliche Zukunft philosophisch akut geworden sei (so 229).

In diesen temporalen Rahmen passt sich nun auch eine der zentralen Passagen Jonas' Überlegungen ein, in denen Diagnose und Lösung, kritische Anamnese und Vorschlag zusammenfinden. Für eine "Ethik der Fernverantwortung" (63) formuliert Jonas einen Imperativ, der in Form und Status bewusst an Kants praktische Philosophie anknüpft. Jonas setzt mit einer Kritik an Kant ein, zumal der kategorische Imperativ selbst nicht moralisch kodiert sei, sondern sich allein aus der Bewahrung logischer Konsistenz ergebe (vgl. 35). Das eigene Handeln demnach vor den virtuellen Richterstuhl verallgemeinerter Maximen zu ziehen und sich dann zu fragen, ob man das wollen könne, bleibe im Formalen stecken. ${ }^{15}$ Denn die Zukunft der Selbstkonsistenz zu opfern, sei in der Kantischen Deontologie gerade nicht ausgeschlossen - und genau dieser Punkt sei für die heutige Zeit virulent geworden, zumal erst in der Gefahr die Pflichten zur Erhaltung im Angesicht des Fortschritts hervortreten (ebd. und 249).

Wiederum Kant folgend bietet Jonas in der Formulierung des sneuen Imperativs unterschiedliche Versionen an. Sie lauten:

»Handle so, daß die Wirkungen deiner Handlung verträglich sind mit der Permanenz echten menschlichen Lebens auf Erden'; oder negativ ausgedrückt: $>$ Handle so, daß die Wirkungen deiner Handlung nicht zerstörerisch sind für die künftige Möglichkeit solchen Lebens;; oder einfach: `Gefährde nicht die Bedingungen für den indefiniten Fortbestand der Menschheit auf Erden'; oder wieder positiv gewendet: 'Schließe in deine gegenwärtige Wahl die zukünftige Integrität des Menschen als Mit-Gegenstand deines Wollens ein.« $(36)^{16}$

\footnotetext{
15 ZurVergewisserung: "Handle nur nach derjenigen Maxime, durch die du zugleich wollen kannst, dass sie ein allgemeines Gesetz werde.«Immanuel Kant, Grundlegung der Metaphysik der Sitten, AA IV, 421.

${ }^{16}$ Jonas meint zugleich, dass dieser mneue Imperativ das einzige Beispiel sei, in dem der Kantische kategorische Imperativ tatsächlich kategorisch sei (so PV, 92).
} 
Das "Für-Immer« hat in Jonas' Imperativ, wie eingangs erwähnt, den Status einer kontrafaktischen Unterstellung. Der »indefinite Fortbestand der Menschheit« münzt die Endlosigkeit zur Erweiterung unserer Verantwortung um, die bereits in den Entscheidungen der Gegenwart liegt. Dieser verantwortungsethische Imperativ setzt demnach nicht mehr auf die logische Konsistenz im kognitiven Haushalt reiner Vernunftwesen, sondern auf die Übereinstimmung unserer Handlungen und deren Folgen mit der Existenz der Menschheit ad infinitum (36f.). Die Zukunft der Menschheit gilt Jonas dabei als erste Pflicht menschlichen Kollektivverhaltens im Zeitalter technischer Zivilisation (so 245) ${ }^{17}$ Jenen Imperativ flankiert Jonas mit einer hermeneutischen Regel des Negativen, nämlich der schlechteren Prognose stets den Vorrang einzuräumen: "Es ist die Vorschrift, primitiv gesagt, daß der Unheilsprophezeiung mehr Gehör zu geben ist als der Heilsprophezeiung." (70)

Dies entspricht der alten CIA-Weisheit, auf das Beste zu hoffen, aber auf das Schlimmste vorbereitet zu sein. Jonas selbst nennt es eine "Heuristik der Furcht« (8 und 392).

Doch diese lebt von einer Zusatzannahme, die Jonas dann auch ausführlich diskutiert, hier aber nur knapp erwähnt werden kann. Denn die Frage ist ja, warum es aus genuin ethischen Gründen ausgeschlossen sein müsse, dass die Menschheit tatsächlich ein Ende finde (36); in anderen Worten: wie sich aus dem ontischen Faktum, dass es die Menschheit gibt, das ontologische Gebot ergebe, dass es sie weiter geben solle (186f.; siehe das gesamte Kap. IV). Dabei setzt Jonas auf drei Gedankengänge. Zum einen auf eine ausholende Kritik des angeblich "garstig breiten Grabens" zwischen Sein und Sollen (bes. 92-94), sodann auf die "dichte Beschreibung « von Fällen nicht-reziproker Verantwortung (etwa bei Kindern - und von dort aus gegenüber der Natur; 84f., 177, 234) und schliesslich auf die weitreichende These vom sittlichen Eigenrecht der Natur (29), wobei diese Auffassung wiederum oszilliert, wenn man sie etwas drängt: nämlich zwischen einer Behauptung über unsere Stellung zur Natur als etwas "Heiligem", d.h. unter keinen Umständen Anzutastendem (393) und der gleichsam post-vitalistischen Sicht, im organischen Leben habe die Natur ihr Interesse kundgetan; das Leben als explizite Konfrontation mit seinem Nichtsein wähle sich immer schon selbst, wie es bereits durch seine pure Existenz manifestiert habe (156f.).

${ }^{17}$ Damit ist das Problem des Freitods berührt, das Jonas auch umfassend behandelt. Dabei hält er den individuellen Suizid für potenziell berechtigt, schliesst dies aber im Blick auf die Menschheit als intrinsischen Wert aus (dazu bes. PV, 36, 80f., 97). 
Auf welch unsicherem Boden Jonas hier steht, lässt er bisweilen selbst durchblicken, um schliesslich fragend anzudeuten, ob sich jener Imperativ zum Sein des Menschen und der Natur überhaupt ohne Metaphysik, ja ohne Religion begründen lasse (8 und 99). Leider ist zu vermuten, dass Jonas hier die falsche Antwort auf ein falsch gestelltes Problem gibt - aber es ist auch zu erwägen, ob unser eigentliches Thema, das »Für-Immer" als kontrafaktische Unterstellung, sich auch unabhängig von jenen metaphysischen und ggf. religiösen "Anfangsgründen« verhandeln lässt. Davon möchte ich hier ausgehen, um hinzuzufügen, dass Jonas'»Heuristik der Furcht» auch andere Formen als die des Imperativen annehmen kann. Zunächst unterstreicht er, dass es nicht um die vom Handeln abratende Furcht gehe, sondern um jene, die zurVerantwortung wesenhaft gehöre und rufe (391). Es gelte, »die verlorene Ehrfurcht vom Schaudern, das Positive vom vorgestellten Negativen zurückgewinnen" (393); doch Ehrfurcht allein reiche nicht aus, zumal das "Gefühl der Verantwortung« (170) nötig sei, gepaart mit einer neuen Art humaner Demut, gerade nicht, weil wir so unfähig wären, sondern mit klarem Blick für das, was wir alles können (55).

Es mag deutlich geworden sein, dass es Jonas nicht an der Emphase letzter Dringlichkeit fehlen lässt, würde er doch die Devise Hölderlins als Ausdruck fehlender Umsicht ad acta legen:

"Nah ist

Und schwer zu fassen der Gott.

Wo aber Gefahr ist, wächst

Das Rettende auch."

Folgt man Jonas' - angeblich (so 46) - anti-utopischer und nichteschatologischer Verantwortungsethik, ist daran bestenfalls die zweite Zeile zutreffend. ${ }^{18}$

\footnotetext{
${ }^{18}$ Hölderlin, Patmos (1802). Hier wird allerdings theologiekritisch mit der Lesart gespielt, dass es gerade Gottes Nähe sei, die jene Gefahr in sich berge. Heideggers spätere Resignativgeste, nach der suns nur noch ein Gott retten könne`, würde demnach die kritische Spitze in Hölderlins Dichtung wieder konservativ entschärfen; siehe Heidegger im Originalton: "Nur noch ein Gott kann uns retten. Die einzige Möglichkeit einer Rettung sehe ich darin, im Denken und Dichten eine Bereitschaft vorzubereiten für die Erscheinung des Gottes oder für die Abwesenheit des Gottes im Untergang." Spiegel-Gespräch mit Martin Heidegger [23. September 1966] «, in: ders., Reden und andere Zeugnisse eines Lebensweges. GA 16, Frankfurt a.M. 2000, 652-683, 671 - mit Bezug auf Hölderlin; auch ebd., 678.
} 


\section{A self-destroying prophecy}

Was mich an Jonas' imperativer Reaktion auf die Untiefen der Moderne interessiert, ist weniger der gegenwartsdiagnostische Befund (der inhaltlich, wohl aber nicht gemäss seiner sorgenvollen Dezibel ein update vertragen könnte). Eher geht es darum zu klären, was hinter der >kontrafaktischen Unterstellung«steckt. Wie funktioniert sie, was macht man (nach Jonas) mit ihr, und wie könnten wir uns diese Figur aneignen?

Was Jonas zunächst zu unterstellen scheint, ist das »Für-Immer» der Menschheit als Gattung. In gewisser Weise dreht er damit das Feuerbachsche Verdikt um, indem nicht Eigenschaften der Gattung ins Divine gesteigert werden, sondern die infinitas Dei auf die nun endlose Gattung angewendet wird. ${ }^{19}$ Dabei ist nicht diese Präsupposition von Eigenwert, sondern hat Gewicht nur mit Blick auf die daraus folgenden Erwägungen, die wiederum in unsere moralisch signifikanten Entscheidungen eingehen sollen. Allerdings, das muss man hinzufügen, bleibt der Status der Unterstellung recht verwaschen. Denn entweder legt man Jonas' Imperativ so aus, dass es um das Fortbestehen der menschlichen Gattung als Ziel geht - dann wären alle Optionen so abzuwägen, dass sie diesem telos approximatorisch zur Verwirklichung verhelfen; oder aber es wird davon ausgegangen, dass die Gattung das Infinite schon in sich trage - um daraufhin alle Handlungsvollzüge zu befragen. Im einen Fall steht das »Für-Immer« für eine Intention, im anderen für eine Gegebenheit. In beiden Szenarien aber kommt es auf die konsequentialistische Note an, die Folgen unseres Handelns im Angesicht ihrer nur schweren oder unmöglichen Revidierbarkeit zu bedenken. ${ }^{20}$ "Für immer« als adverbiale Bestimmung amplifiziert also die Dringlich-

\footnotetext{
$19 \mathrm{Zu}$ den religionskritischen Spitzensätzen, die verraten, dass Feuerbach in die Schule Luthers gegangen ist, dort aber nicht sitzen bleiben wollte, gehört folgender: »Ein Gott allein für sich ist kein Gott - das heisst eben nichts andres als: ein Gott ohne den Menschen ist nicht Gott; wo kein Mensch, ist auch kein Gott; nimmst du Gott das Prädikat der Menschlichkeit, so nimmst du ihm auch das Prädikat der Gottheit; fällt die Beziehung auf den Menschen weg, so fällt auch sein Wesen weg." So Ludwig Feuerbach, Das Wesen des Christentums. Ausgabe in zwei Bänden, hg. v. Werner Schuffenhauer, Berlin 1956, Band II, 540 (teilweise gesperrt im Orig.), vgl. ebd., 320 und 536.

${ }^{20}$ Damit stellen sich die Standardprobleme aller konsequentialistischen Ethiken, nämlich was 'Folgen « sein sollen, wie weit man sie zurückverfolgen muss und wie es um die Verantwortung der in Rede stehenden Personen konkret steht; dazu Dieter Birnbacher, Analytische Einführung in die Ethik, Berlin/New York ${ }^{22007, ~ 177-193 . ~}$
} 
keit jenerVersuchsanordnung und setzt die verheerenden Resultate kursiv.

Das "Für-immer« ist dabei, wie angedeutet, mehr als eine regulative Idee, auch mehr als ein blosses Als-Ob. Es ist deshalb ımehr als all dies, weil beide Alternativen implizieren, nicht real werden zu können, während die Zukünfte, die Jonas sorgenvoll betrachtet, gerade davon leben, unsere Zukunft sein zu können, mit rasant steigender Wahrscheinlichkeit. Es ist aber auch `mehr als das Implikat gesellschaftlicher Institutionen, etwa dem Hochzeitsversprechen (das vollkommen zurecht von der ewigen Liebe ausgeht - alles andere wäre nicht nur hoffnungslos unromantisch, sondern eine Konfusion der Empfindung und der Grammatik!). Zwar wird jenes Versprechen $\mathrm{zu}$ oft gebrochen, als dass der Realitätssinn darüber hinweg gehen könnte (was an jenem "Für- Immer" und dessen Sinn gar nichts ändert); es ist aber Ausdruck der Tiefe der Liebe in der Gegenwart, die Zukunft sein und werden will. Bei Jonas geht es hingegen um eine ganz sichere und damit umso bedrohlichere Zukunft, die durch Entscheidungen im Jetzt als realisierte Zukunft gerade verhindert werden soll.

Neben den konsequentialistischen Zutaten fügt Jonas prozeduralethische bei. Er folgt dabei den Kantischen Vorgaben, zumal auch sein `neuer Imperativ einem Testverfahren gleichkommt: Ging es bei Kant um den Filter, der durch erprobte Verallgemeinerung das Erlaubte aussondert, geht es bei Jonas um eine Prolepse, die uns sagen soll, was mit Bezug auf den »indefiniten Fortbestand der Menschheit" als intrinsischer Wert auszuschliessen ist. Hier gilt also nicht: "Alles ist erlaubt, aber nicht alles dient zum Guten" (I Kor 6,12), sondern was erlaubt ist, dient sehr wohl zum Guten, nämlich dem besagten Telos des als unendlich angenommenen Lebens. Theoretisch betrachtet, kommen in Jonas' Ansatz also konsequentialistische (Folgen), verfahrensethische (Test) und deontologische (intrinsischer Wert) Module zusammen.

Schauen wir uns die Logik jener Unterstellung etwas genauer an. In ihr geht es offenbar nicht um propositionale Unterstellungen in Form von Sätzen und Aussagen, sondern um eine "mögliche Welt» als künftiger Zustand jener, in der wir leben. Kommen wir zunächst zum Problem der Begründung eines solchen Szenarios. Dazu lassen sich verschiedene Wege einschlagen:

(i) probabilistisch: Hier geht es um die Wahrscheinlichkeit des Eintretens des unterstellten Ereignisses, in Jonas' pessimistischer Eschatologie also die (nukleare) Selbstvernichtung der Menschheit. Die mag nur der Endpunkt einer schon einsetzenden Dynamik sein, so- 
dass sich auch hier die bekannte Ellipse zwischen sschon jetzt - aber dann erst recht aufspannt, ${ }^{21}$ d.h. zwischen aktueller Machbarkeit im Technischen und künftiger Erwartbarkeit im noch Ausstehenden (noch einmal: Genau dies trifft für regulative Ideen, Als-ob-Präsuppositionen und wunderbare Hochzeitsversprechen nicht $\mathrm{zu}$ ).

(ii) heuristisch: Hier hingegen geht es nicht um angenommene oder gar berechnete Wahrscheinlichkeiten, sondern um das, was das vorausgesetzte Szenario den Menschen lehrt, indem es ihn etwas sehen lässt. Nicht die Wahrheit des Vorweggenommenen zählt hier, sondern ihre hermeneutische Erschliessungskraft, die rekursiv jene Eingangsvoraussetzungen pragmatisch rechtfertigt.

(iii) holistisch: Schwächer, weil etwas umständlicher, fällt diese dritte Option aus. Sie behauptet, die Annahme eines Szenarios (ein Weltzustand) und die damit verbundene Setzung eines Wertes (Menschheit) stünden im Dienst, einen ganz anderen Wert zu etablieren, der jener szenenhaften Setzung vorgelagert ist: die Solidarität und Achtsamkeit in der Gegenwart. Diese wäre wiederum rekursiv durch eine Negativ-Zukunft begründet, die ihrerseits voraussetzt, dass es das von ihr Begründete gebe. Man mag das als zirkulär abtun, könnte es aber im Gefolge von Wittgenstein auch als ein (holistisches) Netzwerk unterschiedlicher Werte verstehen, die sich gegenseitig halten. ${ }^{22}$

(iv) deutungsmächtig: Eine vollkommen andere Sicht der Dinge geht von der Unvermeidlichkeit zumindest impliziter Vorwegnahmen möglicher Zukünfte aus. Sie unterhielten eine enge Verwandtschaft mit den (tot gesagten) Grosserzählungen, mittels derer wir uns orientieren. Nicht das Dasein dieser Prolepsen und Narrative müsste dann eigens begründet werden, sondern die Entscheidung stünde an, in und mit welchen wir leben können. Das bringt es mit sich, dass die eine Geschichte das Potenzial hat, andere ungewollte Erzählungen zu verdrängen. Das wäre die eher offensive Variante. Die etwas freundlichere wäre diejenige, die von der Evidenz bestimmter Szenarien für unser Selbstverständnis ausginge. In beiden Versionen, derVerdrängung und der Evidenz, sind jedoch Deutungskämpfe am Werk.

${ }^{21}$ Dazu Eberhard Jüngel, Die Welt als Möglichkeit und Wirklichkeit. Zum ontologischen Ansatz der Rechtfertigungslehre, in: ders., Unterwegs zur Sache. Theologische Bemerkungen, München 1972, 206-233, 213.

22 Dazu Avrum Stroll, Why On Certainty Matters, in: Danièle Moyal-Sharrock and William Brenner (eds.), Readings of Wittgenstein's On Certainty, Houndmills/Basingstoke/New York 2005, 33-46. 
Soweit zur Frage der Begründung von Jonas' Imperativ. Ein weiteres Moment, das in jener Order nur geräuschlos mitläuft, ist der Umstand, dass wir es immer noch nicht mit Fakten, sondern Kontrafakten zu tun haben. Es geht nicht um das, "was der Fall ist", sondern darum, was der Fall der Fälle denn sein könnte - und dies nicht in neutraler Indifferenz, sondern innerhalb eines Settings, das seine unbedingte Relevanz für uns in sich trägt und den Ausstieg, nichts $\mathrm{zu}$ entscheiden und sich $\mathrm{zu}$ enthalten, verbietet. Wer aber entscheiden muss, braucht Parameter, Gründe und - insbesondere bei Kontrafakten - Werte. Die Referenz auf die Tatsachen reicht hier nicht mehr aus, sodass evaluative Elemente ins Spiel kommen müssen. ${ }^{23}$ Die kontrafaktischen Szenarien abzuwägen und sich, sie vorwegnehmend, zu entscheiden, setzt einfliessende Werte genauso voraus, wie es Ausdruck dieser sein mag. Jonas' negative Eschatologie gibt dafür selbst ein prominentes Beispiel.

Und ein drittes Moment - neben Begründungs- und Wertfragen - tritt hinzu. Denn zur Logik derVorwegnahme gehört es, dass Prognose und Realisierung, Annahme und Eintreten nicht unverbunden nebeneinander stehen. Was damit angesprochen ist, kursiert unter dem Etikett self-fulfilling prophecy in unterschiedlichen Diskursen. Für solche Prophezeiungen (und ihren Effekt) haben wir ganz unterschiedliche Beispiele. Man denke an den Wetterbericht, der das Wetter gerade nicht beeinflusst - es gibt also kein meteorologisches Placebo. Und man vergleiche diesen Fall mit den Wirtschaftsnachrichten, die in das Markt- und Aktiensystem erheblich eingreifen können. Wie verhält sich dazu die hier besprochene Versuchsanordnung? Jonas: „Denn hier, wo Menschen über Menschen nachdenken, und zwar öffentlich, ändert das Dasein der Theorie als selber geschichtlicher Tatsache die Bedingungen des Erkenntnisgegenstandes." (209f.; im Orig. kursiv)

Demnach liegt der hier betrachtete Fall näher an den Wirtschaftsnachrichten als am Wetterbericht; der Zuschnitt des theoretischen Rahmens interferiert mit dem, was mit seiner Hilfe beschrieben wird. Doch dabei, wir haben es mehrfach gesehen, liegen nicht nur deskriptive Terme vor (der Zustand der Menschheit und ihre Zukunft), fliessen doch normative Voraussetzungen in die Rechnung

\footnotetext{
${ }^{23}$ Das hat vor allem der schon erwähnte Olaf L. Müller im Kontext eines "pragmatischen Pazifismus" deutlich gemacht. Neben dem in Anm. 6 genannten Text siehe auch: Pazifismus mit offenen Augen, in: Der gerechte Friede zwischen Pazifismus und gerechtem Krieg. Paradigmen der Friedensethik im Diskurs, hg. v. JeanDaniel Strub und Stefan Grotefeld, Stuttgart 2007, 23-59; ders., Pragmatist Pacifism, in: Politics of Pacifism: Commandment - Ideal - Impossibility? Theological Contributions to Peace Ethics, hg. v. Hartmut von Sass (2016; im Druck).
} 
bereits mit ein (der unbedingte Wert der Menschheit und ihre bedrohte Zukunft). Auch Jonas setzt also auf die Beeinflussung der Realisierung von $x$ durch dieVoraussage von $x$ - nur mit umgekehrtem Vorzeichen. Es geht nicht um die Steigerung der Wahrscheinlichkeit, dass $x$ verwirklicht werde, sondern die Prognose dient der Dezimierung oder gar Verhinderung von $x$. Während die Gewinnprognose für einen Konzern den effektiven Profit steigern oder gar generieren kann, damit die Prognose womöglich noch übertroffen werde, kann die Annahme der Zerstörung der Menschheit offensichtlich nichts dergleichen ausrichten - und dennoch wirksam sein, nämlich als self-destroying prophecy. Wie ein Geheimnis, das man verrät, besteht hier die Hoffnung oder vielleicht gar die Erwartung darin, dass ihre Offenlegung in die eigene Revision münde.

Es ist deutlich zu sehen: Selbst der wohl schärfste Kritiker des Prinzips Hoffnung hofft ungemein prinzipiell, wodurch auch hier ein eschatologischer Horizont, allerdings ohne "Vorbehalt" aufgespannt wird. Eine fürchterliche Zukunft wird hier vorweg genommen, um ihren heilsamen Dienst an der Gegenwart zu vollführen - dies folgt offenbar einer etwas anderen Blickrichtung, als es das gut hermeneutische "Zukunft braucht Herkunft" getan hat (Odo Marquard).

\section{Coda: ein Vorschlag}

Gegen Ende möchte ich nun auf jene genannte Frage zurückkommen, wie wir uns Jonas' Imperativ aneignen können, was wir also mit solchen Szenarien 'probehalber moralisch tun können. Nun ist mehrfach festgestellt worden, dass Jonas die doppelte Verantwortung als für jemanden und vor jemandem auf die ersteVersion konzentriert. ${ }^{24}$ Öffnet man diese Reduktion wieder auf jene Dopplung, mag neben der Handlungsrelevanz gegenwärtiger Entscheidungen unser Orientierungssinn stärker hervortreten, der sich hier Gehör verschafft. Dann geht es nicht primär um Fragen des richtigen und moralisch rational begründeten Handelns, sondern um die umfassendere Frage, wie wir uns vor anderen und uns selbst verstehen möchten. ${ }^{25}$ Jonas' Imperativ, die Effekte unseres Handelns gedanklich vorweg-

\footnotetext{
${ }^{24}$ So etwa Wolfgang Huber, Kirche in der Zeitenwende. Gesellschaftlicher Wandel und Erneuerung der Kirche, Gütersloh (1998) ${ }^{3} 1999$, 182f. - mit Verweis auf PV 36 und 189.

${ }^{25}$ Vgl. Johannes Fischer, Leben aus dem Geist. Zur Grundlegung christlicher Ethik, Zürich 1994, 137-142; ders., Verstehen statt Begründen. Warum es in der Ethik um mehr als nur um Handlungen geht, Stuttgart 2012, bes. $69 \mathrm{f}$.
} 
zunehmen, ähnelt analogen, teils spielerischen, teils gerade im Spiel toternsten Fragen: 'Stelle Dir vor, dieser Mensch würde nicht mehr da sein (; ) Gehe davon aus, dass Du dies noch unzählige Male wiederholen müsstest! ( (cf. Nietzsche); $>$ Nimm an, dass Du nur noch ein halbes Jahr zu leben hättest - was machst Du dann?<; oder eben mit Jonas: Setze voraus, dass diese Entscheidung nicht mehr zurückzunehmen ist und ihr Resultat sewig gilt - wie wirst Du Dich nun verhalten?.

Jonas kommt das Verdienst zu, diesen hypothetischen Imperativen - als Invitativen - neue Betätigungsfelder zugeordnet zu haben. Doch verhandelt er sie noch in einem klassischen Rahmen, der auf Begründen setzt, nicht auf Verstehen, mithin auf metaphysische Letztbegründung, nicht auf die Artikulation von Orientierung, Selbstverständnis und dessen, was uns als Menschen wichtig ist. Übt man diesen "fröhlichen Wechsel« ein, entlastet man jenen Befehl von unnötigen Beweispflichten, müsste sich aber auch einer zarteren, verletzlicheren Sprache bedienen, um dem, was sich in der Beantwortung jener existentiellen Fragen aussprechen könnte, auf die Spur zu kommen. Nun wird nichts mehr begründet und argumentativ etabliert, sondern es würde zaghaft, vielleicht erzählerisch bekundet, was mich berührt, für mich wichtig ist, weil es etwas mit mir zu tun hat. Die Antworten, die ich auf jene Fragen geben würde, sagten, wie ich mich verstehen kann und wer ich für andere und vor mir selbst eigentlich sein möchte. Diese Art ethischer Nachdenklichkeit führt nichts Neues durch vermeintliche Deduktionen ein, sondern versucht zu entfalten, was uns (unbedingt?) angeht. Sie stiftet nicht in ohnehin verspäteter Gebärde unseren Zugang zu dem, wovon wir längst umgeben sind, sondern geht davon aus, dass wir mit all dem auf eine bestimmte Weise, jedenfalls in mitunter "starken Wertungen" schon verbunden sind. ${ }^{26}$ Diese Ethik gibt nicht(s) vor, sondern sie legt aus; und Auslegungen sind gerade nicht frei vom Anspruch, normativ zu sein, sondern eine eigene Weise, Normativität geltend zu machen.

Einer dieser hypothetischen Invitative könnte lauten: >Stell Dir vor, Du dürftest für einen Tag Lemmy Kilmister oder David Bowie sein - was machst Du? .......

- Hartmut von Sass ist Privatdozent für Systematische Theologie und Religionsphilosophie an der Universität Zürich, stellvertretender Direktor des Collegium Helveticum von Universität und ETH Zürich sowie Leiter des Ludwik-Fleck-Zentrums für Wissenschaftstheorie.

${ }^{26}$ Dazu Charles Taylor, Self-interpreting animals, in: ders., Human Agency and Language. Philosophical Papers 1 [1985], Cambridge 22005, 45-76, 65. 